AEI-1999-38

hep-th/9911170

\title{
Yang-Mills Integrals
}

\author{
Werner Krauth $\ddagger$ Jan Plefka $\S$ and Matthias Staudacher $\S$ \\ $\ddagger$ CNRS-Laboratoire de Physique Statistique, Ecole Normale Supérieure, 24, rue \\ Lhomond, F-75231 Paris Cedex 05, France \\ $\S$ Max-Planck-Institut für Gravitationsphysik, Albert-Einstein-Institut, Am \\ Mühlenberg 1, D-14476 Golm, Germany
}

\begin{abstract}
S U(N)$ Yang-Mills integrals form a new class of matrix models which, in their maximally supersymmetric version, are relevant to recent non-perturbative definitions of 10-dimensional IIB superstring theory and 11-dimensional M-theory. We demonstrate how Monte Carlo methods may be used to establish important properties of these models. In particular we consider the partition functions as well as the matrix eigenvalue distributions. For the latter we derive a number of new exact results for $S U(2)$. We also report preliminary computations of Wilson loops.

PACS numbers: 11.15.-q,11.25.-w,12.60.Jv
\end{abstract}

Submitted to: Class. Quantum Grav.

I Talk presented by M. Staudacher at Strings '99, Potsdam, July 19-24 1999 


\section{Motivation}

Recently there has been considerable interest in dimensionally reduced Yang-Mills theories as a means to obtain non-perturbative information on superstring theory and M-theory. The possible relevance of these systems to quantum gravity appeared in [1] through the light cone quantization of the 11-dimensional supermembrane. It was argued in [1] that 10-dimensional $S U(N)$ super Yang-Mills theory reduced to one dimension i.e. matrix quantum mechanics - correctly quantizes the supermembrane in the large $N$ limit. The very same system, first studied (without reference to applications to quantum gravity) in [2], has recently been interpreted as a non-perturbative attempt at M-theory [3]. Unfortunately, on the technical side, very little is known about this model: It is suspected [1], [3] that a novel, intricate large $N$ limit is required, but only few concrete results are available. This motivates the study of a simpler system: The complete reduction of Yang-Mills theory to $0+0$ dimensions. In addition, the reduction of the ten-dimensional susy gauge theory path integral to a matrix integral has been at the heart of an alternative proposal to directly define non-perturbative IIB string theory [4]. More generally we may study the complete reduction of D-dimensional $S U(N)$ YangMills theory. Then the path integral of the field theory simplifies to an integral over the group's Lie algebra, with a flat measure: a Yang-Mills integral. Denoting the gauge potential by $X_{\mu}^{A}$ and their superpartners by $\Psi_{\alpha}^{A}$ we obtain

$$
\mathcal{Z}_{D, N}^{\mathcal{N}}:=\int \prod_{A=1}^{N^{2}-1}\left(\prod_{\mu=1}^{D} \frac{d X_{\mu}^{A}}{\sqrt{2 \pi}}\right)\left(\prod_{\alpha=1}^{\mathcal{N}} d \Psi_{\alpha}^{A}\right) \exp [-S(X, \Psi)] .
$$

with the Euclidean "action"

$$
S(X, \Psi)=-\frac{1}{2} \operatorname{Tr}\left[X_{\mu}, X_{\nu}\right]\left[X_{\mu}, X_{\nu}\right]-\operatorname{Tr} \Psi_{\alpha}\left[\Gamma_{\alpha \beta}^{\mu} X_{\mu}, \Psi_{\beta}\right] .
$$

The a priori allowed dimensions for the reduced supersymmetric gauge theory are $D=3,4,6,10$ corresponding to $\mathcal{N}=2,4,8,16$ real supersymmetries. For the bosonic, i.e. the non-supersymmetric case $\mathcal{N}=0$, we omit the Grassmann variables $\Psi_{\alpha}^{A}$, and may study all dimensions $D \geq 2$.

There are numerous further reasons for being interested in the integrals eq.(可):

- The susy integrals are crucial for the computation of the Witten index of the above mentioned quantum mechanical gauge theories, as they contribute to the so-called bulk part of the index [5] (cf also [6] for an earlier calculation).

- In the maximally supersymmetric case, the system describes to leading order the statistical distribution of a system of $N$ D-instantons (or "-1-branes").

- The $\mathcal{N}=16$ integral appears in very recent work developing a multi-instanton calculus for the $\mathcal{N}=4, D=4 S U(\infty)$ conformal gauge theory [7], and again in the large $N$ limit of $\operatorname{Sp}(N)$ and $\mathrm{SO}(N) \mathcal{N}=4$ susy gauge theory [8].

- Finally we can regard the integrals eq.(1) as a version of the Eguchi-Kawai reduced gauge theory. The original work [9] focused on a lattice formulation and employed unitary matrices, while the above integrals use the hermitean gauge connections $X_{\mu}^{A}$. 
This is similar to [10]; however, we apply neither gauge fixing nor quenching prescriptions to the above integrals. The interesting question is whether the models eq.(1) encode universal information on the full gauge field theory as $N \rightarrow \infty$.

The integrals of eq.(四) appear to be singular due to the "valleys" of the action, i.e. the directions in the configuration space of the $X_{\mu}^{A}$ where all $D$ matrices commute. Recent work has however proven this intuition wrong: Yang-Mills integrals do exist in many interesting cases.

\section{Partition functions: Convergence properties and some exact results}

Indeed, the rigorous results of [5] (see also [6]) show that for the gauge group $S U(2)$ the susy integrals converge in dimensions $D=4,6,10$. The calculations are easily repeated for the bosonic case [1], and the convergence condition $D \geq 5$ is found. Unfortunately, no rigorous methods exist to date for higher rank gauge groups $N \geq 3$.

In [11], [12], [13] we developed methods to numerically test convergence of singular multidimensional integrals. The idea is to perform a Metropolis random walk weighted by the integrand, and to merely measure the autocorrelation function of subsequent configurations. In this approach, a unit autocorrelation function signals the presence of a nonintegrable singularity.

As an illustration we plot in Fig.1 the autocorrelation function of the $S U(2)$ bosonic integrals.
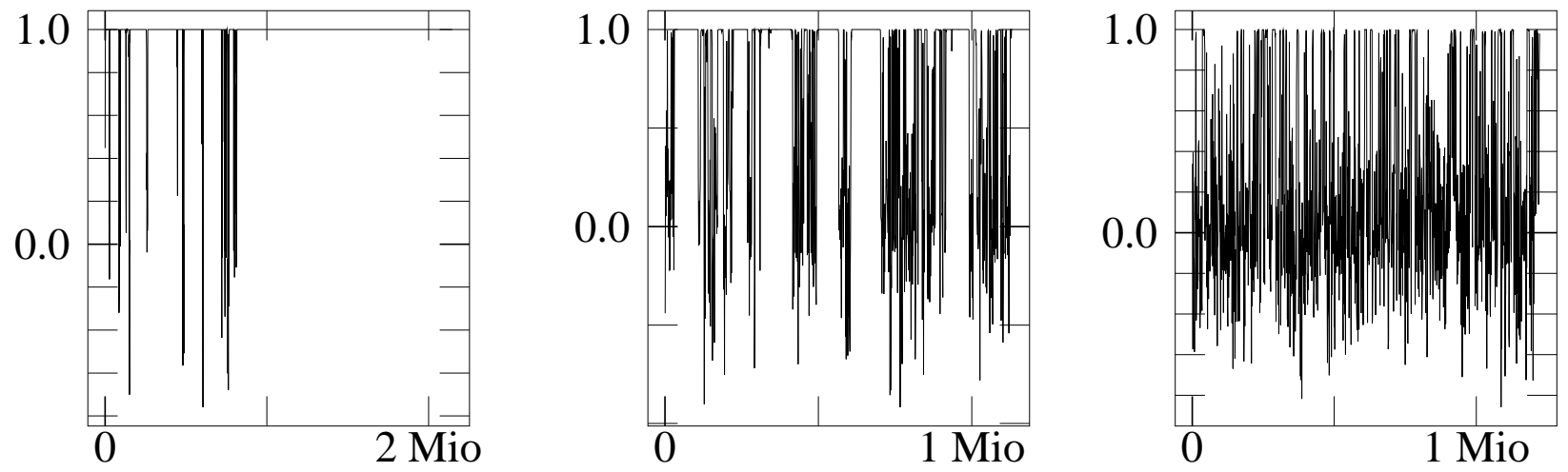

Fig. 1 Autocorrelation functions versus Monte Carlo time for the $S U(2)$ bosonic integral with, from the left, $D=3,4,5$.

We are clearly able to reproduce the convergence condition $D \geq 5$ : For $D=5$ the configurations decorrelate well and the whole integration space is properly sampled. (One observes increasingly improved decorrelation for $D=6,7, \ldots$, not shown in Fig.1.) In contrast for $D=3$ the system gets quickly trapped in a singular configuration: The Markov chain gets lost in a valley, and the integral is divergent. $D=4$ shows marginal divergence, which agrees with the exact analytical results. 
Applying the same method to higher rank bosonic models, and to the supersymmetric models, we are able to map out the convergence conditions for the Yang-Mills integrals. They read

$$
\begin{aligned}
& D=4,6,10 \quad \text { and } \quad N \geq 2\} \quad \text { for } \quad \mathcal{N}>0 \\
& \left.\begin{array}{lll}
D=3 & \text { and } & N \geq 4 \\
D=4 & \text { and } & N \geq 3 \\
D \geq 5 & \text { and } & N \geq 2
\end{array}\right\} \text { for } \mathcal{N}=0
\end{aligned}
$$

In particular the $D=3$ susy integral is divergent (see [11], [12], [13] for a more detailed discussion of this point).

It would be nice to have a rigorous mathematical proof of the conditions (3). Some understanding may be gained by considering one-loop perturbative estimates of the integrals eq.(11). One has for the supersymmetric case [14]

$$
\begin{aligned}
\mathcal{Z}_{D, N}^{\mathcal{N}} \sim \int \prod_{i, \mu}^{N, D} d x_{\mu}^{i} & {\left[\prod_{\mu} \delta\left(\sum_{i} x_{\mu}^{i}\right)\right] \times } \\
& \times \sum_{G \text { : maximal tree }} \prod_{(i j): \text { link of } G} \frac{1}{\left(x^{i}-x^{j}\right)^{3(D-2)}}+\ldots
\end{aligned}
$$

where the $x_{\mu}^{i}$ are the diagonal components of the matrices $X_{\mu}^{A}$, while it is found in [15] that the bosonic integrals are approximated by

$$
\mathcal{Z}_{D, N}^{\mathcal{N}=0} \sim \int \prod_{i, \mu}^{N, D} d x_{\mu}^{i}\left[\prod_{\mu} \delta\left(\sum_{i} x_{\mu}^{i}\right)\right] \prod_{i<j} \frac{1}{\left(x^{i}-x^{j}\right)^{2(D-2)}} .
$$

Powercounting for large separations $\left(x^{i}-x^{j}\right)^{2}$ yields precisely eq.(3). However, it should be stressed that this does not prove the convergence conditions, since one has to worry about configurations where some separations are small, so that the one-loop approximation becomes invalid.

In the supersymmetric case the value for the integrals is believed to be known:

$$
\mathcal{Z}_{D, N}^{\mathcal{N}}=\frac{2^{\frac{N(N+1)}{2}} \pi^{\frac{N-1}{2}}}{2 \sqrt{N} \prod_{i=1}^{N-1} i !}\left\{\begin{array}{rrr}
\frac{1}{N^{2}} & D=4, & \mathcal{N}=4 \\
\frac{1}{N^{2}} & D=6, & \mathcal{N}=8 \\
\sum_{m \mid N} \frac{1}{m^{2}} & D=10, & \mathcal{N}=16
\end{array}\right.
$$

The $\mathcal{N}=16$ expression was conjectured by Green and Gutperle [16] based on a calculation of the D-instanton effective action of the superstring. A derivation of the terms to the right of the curly bracket in eq.(6)), based on cohomological deformation techniques, was given in [17]. This calculation has still an important loophole (see comments in [13]). However, the formula eq.(6) was numerically checked by Monte Carlo techniques in [11, [12] up to $N \sim 5$. 
For the bosonic case no exact value of the partition function is known except for $S U(2)$ [1], where the result reads

$$
\mathcal{Z}_{D, 2}=2^{-\frac{3}{4} D-1} \frac{\Gamma\left(\frac{D}{4}\right) \Gamma\left(\frac{D-2}{4}\right) \Gamma\left(\frac{D-4}{4}\right)}{\Gamma\left(\frac{D}{2}\right) \Gamma\left(\frac{D-1}{2}\right) \Gamma\left(\frac{D-2}{2}\right)} \quad \text { for } \quad D \geq 5
$$

It would be exciting to find the generalization of this result to higher rank gauge groups - this is after all the "zero-mode" contribution to the Yang-Mills partition function on a D-dimensional torus.

\section{Eigenvalue densities: Asymptotics and the exact $S U(2)$ densities}

Let us shift attention from the partition functions to the correlation functions of the models. The simplest correlators are $S U(N)$ invariant one-matrix correlators: the moments $\left\langle\operatorname{Tr} X_{D}^{k}\right\rangle$ of one matrix, say the $D$-th: $X_{D}$. These are directly related to the distribution of eigenvalues of the matrix: If the eigenvalues of $X_{D}$ are $\lambda_{1}, \ldots, \lambda_{N}$, the eigenvalue density is defined for all $N$ as

$$
\rho(\lambda)=\left\langle\frac{1}{N} \sum_{i=1}^{N} \delta\left(\lambda-\lambda_{i}\right)\right\rangle
$$

The non-zero moments of $\rho(\lambda)$ are then given by

$$
\left\langle\frac{1}{N} \operatorname{Tr} X_{D}^{2 k}\right\rangle=\int_{-\infty}^{\infty} d \lambda \rho(\lambda) \lambda^{2 k}
$$

In an ordinary Wigner type matrix model all moments exist. Yang-Mills integrals are more intricate. Analytical calculations have only been performed for $S U(2)$, and we found the following surprising results. In the $D=4$ susy integral all moments are infinite, even though the integral itself exists, as argued above. In the $D=6$ susy integral the first two moments are finite and one finds

$$
\left\langle\operatorname{Tr} X_{D}^{2}\right\rangle_{D=6}=\frac{1}{2} \sqrt{\frac{2}{\pi}} \quad\left\langle\operatorname{Tr} X_{D}^{4}\right\rangle_{D=6}=\frac{25}{64}
$$

while all higher moments diverge. For the $D=10$ susy integral we have exactly twelve finite moments which are

$$
\begin{array}{rlrl}
\left\langle\operatorname{Tr} X_{D}^{2}\right\rangle_{D=10} & =\frac{8}{25} \sqrt{\frac{2}{\pi}} & \left\langle\operatorname{Tr} X_{D}^{4}\right\rangle_{D=10} & =\frac{9}{80} \\
\left\langle\operatorname{Tr} X_{D}^{6}\right\rangle_{D=10}=\frac{3}{32} \sqrt{\frac{2}{\pi}} & \left\langle\operatorname{Tr} X_{D}^{8}\right\rangle_{D=10}=\frac{297}{4096} \\
\left\langle\operatorname{Tr} X_{D}^{10}\right\rangle_{D=10}=\frac{1089}{8192} \sqrt{\frac{2}{\pi}} & \left\langle\operatorname{Tr} X_{D}^{12}\right\rangle_{D=10}=\frac{184041}{655360}
\end{array}
$$

It would be interesting to find a geometrical or combinatorial interpretation for these numbers. Which densities give rise to this convergence behavior? For $S U(2)$ we can go farther and find the exact densities:

$$
\rho_{D=4}^{\mathrm{SUSY}}(\lambda)=\frac{3 \cdot 2^{5 / 4}}{\sqrt{\pi}} \lambda^{2} U\left(\frac{5}{4}, \frac{1}{2}, 8 \lambda^{4}\right)
$$




$$
\begin{aligned}
\rho_{D=6}^{\mathrm{SUSY}}(\lambda)=\frac{105}{2^{3 / 4} \sqrt{\pi}} \lambda^{2}\left[U \left(\frac{9}{4},\right.\right. & \left.\left.\frac{1}{2}, 8 \lambda^{4}\right)-\frac{33}{16} U\left(\frac{13}{4}, \frac{1}{2}, 8 \lambda^{4}\right)\right] \\
\rho_{D=10}^{\mathrm{SUSY}}(\lambda)=\frac{1287}{64 \cdot 2^{3 / 4} \sqrt{\pi}} \lambda^{2} & {\left[546 U\left(\frac{17}{4}, \frac{1}{2}, 8 \lambda^{4}\right)\right.} \\
& -147 \frac{17 \cdot 19}{8} U\left(\frac{21}{4}, \frac{1}{2}, 8 \lambda^{4}\right) \\
& +45 \frac{17 \cdot 19 \cdot 21 \cdot 23}{256} U\left(\frac{25}{4}, \frac{1}{2}, 8 \lambda^{4}\right) \\
& \left.-\frac{17 \cdot 19 \cdot 21 \cdot 23 \cdot 25 \cdot 27}{2048} U\left(\frac{29}{4}, \frac{1}{2}, 8 \lambda^{4}\right)\right]
\end{aligned}
$$

where $U$ is the Kummer- $U$ function defined as

$$
U(a, b, z)=\frac{1}{\Gamma(a)} \int_{0}^{\infty} d t t^{a-1}(1+t)^{b-a-1} e^{-z t}
$$

Now we see that the above finiteness properties of the moments result from a rather curious powerlike behavior of the densities at large values of $\lambda$. We have for $\lambda \rightarrow \infty$

$$
\rho_{D}^{\mathrm{SUSY}}(\lambda) \sim \begin{cases}\lambda^{-3} & D=4 \\ \lambda^{-7} & D=6 \\ \lambda^{-15} & D=10\end{cases}
$$

This power-like behavior is very different from Wigner type systems where the fall-off at infinity is at least exponential. For the $D$-dimensional bosonic models the density can be worked out as well albeit less explicitly than in eqs.(12), and one finds the asymptotic behavior $\rho_{D}(\lambda) \sim \lambda^{3-D}$.

Moving on to higher values of $N$, we are unable to analytically calculate the eigenvalue densities with presently known techniques. We can, however, find numerically exact densities using Monte Carlo methods. In Fig.2 we illustrate this by plotting the $N=2,3,4 D=4$ susy half-densities (we only plot the $\lambda \geq 0$ part since the densities are symmetric functions). In the $S U(2)$ case the exact expression of eq.(12) and the Monte Carlo data cannot be separated on the scale of the figure.

Now we would like to know how the $S U(2)$ result eq.(14) generalizes to other values of $N$. It is impossible to extract the asymptotics from histograms such as Fig.2, since the tails comprise only a small number of samples. Instead, we can go back to the Markovchain technique of the last section and measure the finiteness of the moments eq.(9) for various values of $N$. We find that for the susy integrals the qualitative behavior of the $S U(2)$ case persists: In the $D=4,6,10$ integrals only the first, respectively, $0,2,12$ moments are finite. We thus conclude, in view of eq.(9), that the asymptotic behavior eq.(14) is valid for all $N$. This is very different from Wigner type random matrix models, where as $N$ increases, the density condenses onto a compact interval. At the sharply defined (at $N=\infty$ ) edge of the interval Wigner distributions show universal behavior. We have argued that in susy Yang-Mills matrix models no such edge exists, indicating that the large $N$ physics of these models is indeed very different. 

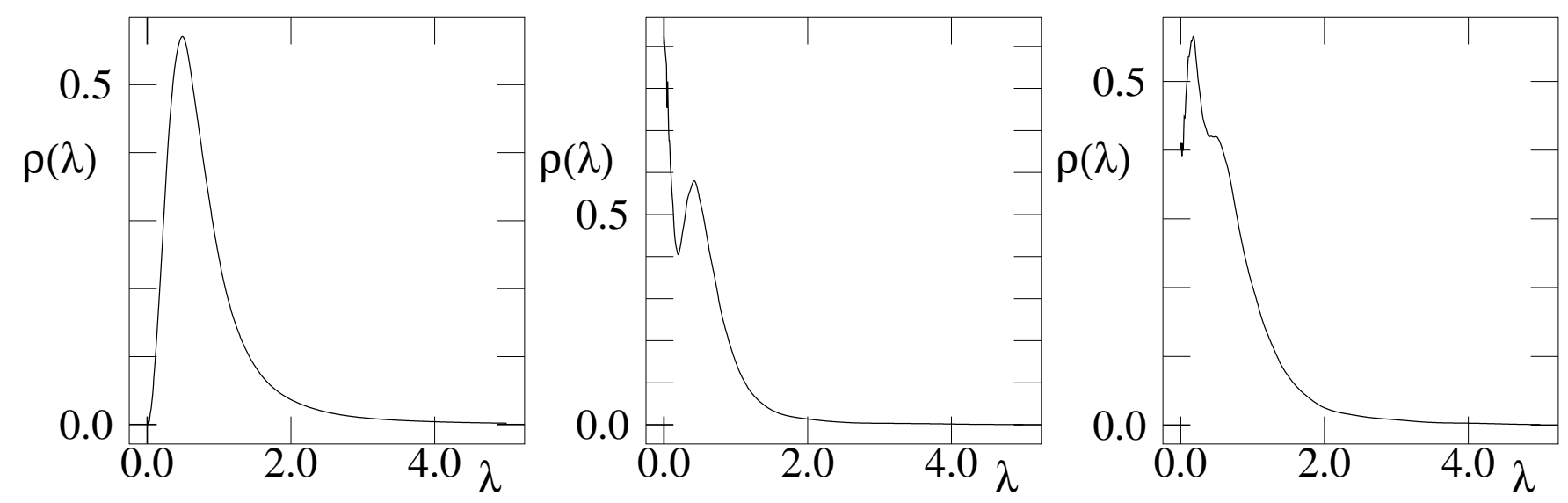

Fig. 2 Eigenvalue (half-)densities for susy $D=4$, from the left, $N=2,3,4$

Let us furthermore compare supersymmetric and non-supersymmetric Yang-Mills integrals. How does the asymptotic behavior of the density eq.(14) change in the absence of susy? We have already mentioned above that for $S U(2)$ this behavior is powerlike as well. Actually we can guess a general formula by looking once more at the effective oneloop estimate of eq.(5). For one-matrix correlators the most dangerous configuration stems from pulling away one coordinate $x_{D}^{i}$ from a bulk configuration of all other $D-1$ coordinates. Powercounting leads to the guess

$$
\rho_{D}(\lambda) \sim \lambda^{-2 N(D-2)+3 D-5} \quad \text { where } \quad N>\frac{D}{D-2}
$$

The same procedure applied to the susy estimate eq.(4) reproduces eq.(14). We then verified the validity of eq.(15) by the same Monte Carlo random walk procedure as above, measuring the finiteness of moments. We thus notice a marked difference to the susy situation: In the bosonic case all moments exist as $N \rightarrow \infty$ for all $D \geq 3$. In particular we expect the eigenvalue distribution to condense onto a compact support, much like for Wigner type models.

\section{Wilson loops: Preliminary Results}

A further natural set of correlation functions of Yang-Mills matrix integrals are Wilson loops. Due to the Eguchi-Kawai mechanism [9], [10], one naively expects them to correspond at $N=\infty$ to Wilson loops in the unreduced gauge field theory. In the proposal of [4] for a non-perturbative definition of the IIB superstring, they have been interpreted as string creation operators [18].

Despite the dimensional reduction of the field theory to zero dimensions we are still able to define an infinite set of independent Wilson loops dependent on an arbitrary contour $\mathcal{C}$ in $D$-dimensional Euclidean space:

$$
\mathcal{W}(\mathcal{C})=\left\langle\frac{1}{N} \mathcal{P} \operatorname{Tr} e^{i \oint_{\mathcal{C}} d y_{\mu} X_{\mu}}\right\rangle
$$


Due to the non-commutative nature of the connections $X_{\mu}$ and the path-ordering $\mathcal{P}$, this is a non-trivial functional of the contour $\mathcal{C}$ despite the fact that the $X_{\mu}$ are spacially constant. In the special case of a rectangular contour with lengths $L$ and $T$ in the $\left(y_{1}, y_{D}\right)$ plane this simplifies to

$$
\mathcal{W}(L, T)=\left\langle\frac{1}{N} \operatorname{Tr} e^{i L X_{1}} e^{i T X_{D}} e^{-i L X_{1}} e^{-i T X_{D}}\right\rangle
$$

We would like to understand how the loops $\mathcal{W}$ behave as a function of $N$ and as a functional of the shape of the contours, in particular whether planar loops satisfy an area-law. We would also like to see whether there are any telltale differences between the supersymmmetric and the bosonic loops.

In the previous sections we have shown how a number of exact results may be derived for the simplest gauge group $S U(2)$. We have not been able to analytically calculate a Wilson loop for an Yang-Mills integral even for $S U(2)$. On the other hand it is possible to obtain high precision numerical results for low values of $N$. In Fig.3a we plot the Wilson loop for a square $(L=T)$ in the case of susy $D=4$ as a function of $L$ for various values of $N$.
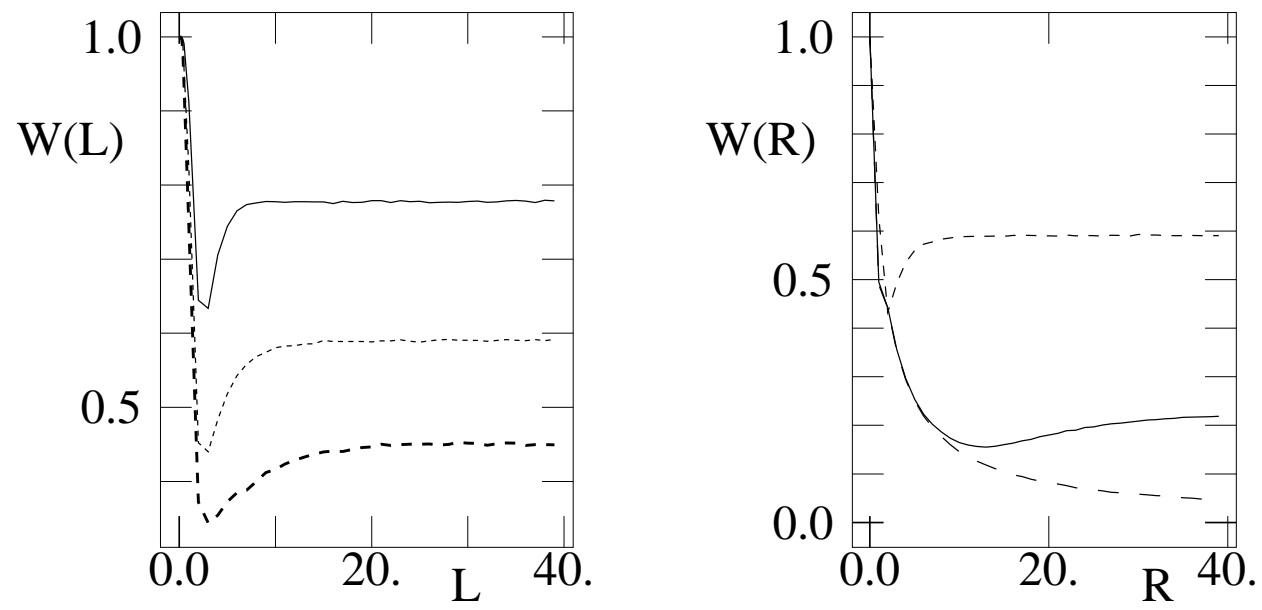

Fig. $3 D=4$ Susy Wilson loops (a) Square of side length $L$ with $N=2,4,8$ (top to bottom) (b) square, regular 16-gon and 64-gon enclosed in circle of radius $R$ with $N=4$.

The behavior for small area is easy to understand. Indeed, for an arbitrary planar loop enclosing a small area of size $\mathcal{A}$, it is straightforward to show, using Stokes' theorem, that $\mathcal{W}(\mathcal{C})=1+\frac{1}{2 N} \mathcal{A}^{2}\left\langle\operatorname{Tr}\left[X_{1}, X_{2}\right]^{2}\right\rangle+\mathcal{O}\left(X^{6}\right)$. This immediately gives

$$
\mathcal{W}(\mathcal{C})=\left\{\begin{array}{cc}
1-\frac{1}{4 N} \frac{N^{2}-1}{D-1} \mathcal{A}^{2}+\ldots & \mathcal{N}=0 \\
1-\frac{1}{2 N} \frac{N^{2}-1}{D} \mathcal{A}^{2}+\ldots & \mathcal{N}>0
\end{array}\right.
$$

and agrees to high precision with the numerical data. A very curious feature of Fig.3a is that the loops tend to a constant for large area. The existence of this constant can be demonstrated analytically. It should be considered a finite $N$ artifact for the following reasons: (a) The constant decreases with $N$, as seen in Fig.3a. (b) The constant depends 
in various ways on the shape of the contour. We checked that by distorting the rectangle to a slightly irregular quadrangle the constant drops to zero for all $N$ as the size increases. In Fig3.b we show various regular polygons approximating a circle of radius $R$ : As we increase the number of edges the constant goes to zero for large $R$.

It is clear from the mentioned features of the Wilson loops that there is an area law neither for very small nor very large areas. An intermediate region in which an area law holds might still be present. We checked, by going to rather larger $N$, that this is not the case for the bosonic models. There has been an interesting suggestion [19] that such an intermediate region may exist for the $D=4$ supersymmetric model.

\section{Conclusions and outlook}

We showed how numerical Markov chain methods can be used to verify non-perturbative convergence conditions for Yang-Mills integrals with and without supersymmetry. The same methods may be applied to establish the convergence properties of correlation functions. Applying the technique to invariant correlators of a single matrix, we are able to accurately predict the asymptotic behavior of the eigenvalue density of YangMills matrix models. The results demonstrate an unusual powerlaw behavior which, in the supersymmetric cases, persists for large $N$. This indicates that the large $N$ limit of these "new" matrix models might indeed be very different from the one of the "old" Wigner type models. We also demonstrated that Monte Carlo methods are capable to rather accurately compute various quantities relevant to these models such as partition functions, correlation functions, spectral distributions and Wilson loops. As opposed to Yang-Mills quantum mechanics [2], [1], [3] we are confronted to a system which allows some non-perturbative analysis, at least for finite $N$.

Yang-Mills integrals are thus an ideal laboratory for exploring new large $N$ techniques. Powerful analytical methods will have to be developed if we are to verify or, maybe more importantly, if we are to bring to good use the ideas presented in [1], [3], [4].

\section{Acknowledgements}

This work was supported in part by the EU under Contract FMRX-CT96-0012.

\section{References}

[1] B. de Wit, J. Hoppe and H. Nicolai, On the Quantum Mechanics of Supermembranes, Nucl. Phys. B305 (1988) 545.

[2] M. Claudson and M. Halpern, Supersymmetric Ground State Wave Functions, Nucl. Phys. B250 (1985) 689; M. Baake, M. Reinecke and V. Rittenberg, Fierz Identities for Real Clifford Algebras and the Number of Supercharges, J. Math. Phys. 26 (1985) 1070; R. Flume, On Quantum Mechanics with Extended Supersymmetry and Non-abelian Gauge Constraints, Annals Phys. 164 (1985) 189.

[3] T. Banks, W. Fischler, S. Shenker and L. Susskind, M-theory as a Matrix Model: A Conjecture, Phys. Rev. D55 (1997) 5112, hep-th/9610043. 
[4] N. Ishibashi, H. Kawai, Y. Kitazawa and A. Tsuchiya, A Large N Reduced Model as Superstring, Nucl. Phys. B498 (1997) 467, hep-th/9612115.

[5] P. Yi, Witten Index and Threshold Bound States of D-Branes, Nucl. Phys. B505 (1997) 307, hep-th/9704098; S. Sethi and M. Stern, D-Brane Bound State Redux, Commun. Math. Phys. 194 (1998) 675, hep-th/9705046.

[6] A.V. Smilga, Witten Index Calculation in Supersymmetric Gauge Theory, Yad. Fiz. 42 (1985) 728, Nucl. Phys. B266 (1986) 45; A.V. Smilga, Calculation of the Witten Index in Extended Supersymmetric Yang-Mills Theory, (in Russian) Yad. Fiz. 43 (1986) 215.

[7] N. Dorey, T.J. Hollowood, V.V. Khoze, M.P. Mattis, and S. Vandoren, Multi-Instantons and Maldacena's Conjecture, JHEP 9906 (1999) 023, hep-th/9810243; Multi-Instanton Calculus and the AdS/CFT Correspondence in $\mathcal{N}=4$ Superconformal Field Theory, Nucl. Phys. B552 (1999) 88, hep-th/9901128.

[8] T.J. Hollowood, V.V. Khoze and M.P. Mattis, Instantons in $\mathcal{N}=4 S p(N)$ and $S O(N)$ theories and the AdS/CFT correspondence, hep-th/9910118.

[9] T. Eguchi and H. Kawai, Reduction of dynamical degrees of freedom in the large $N$ gauge theory, Phys. Rev. Lett. 48 (1982) 1063.

[10] D.J. Gross and Y. Kitazawa, A Quenched Momentum Prescription for Large $N$ Theories, Nucl. Phys. B206 (1982) 440.

[11] W. Krauth, H. Nicolai and M. Staudacher, Monte Carlo Approach to M-Theory, Phys. Lett. B431 (1998) 31, hep-th/9803117.

[12] W. Krauth and M. Staudacher, Finite Yang-Mills Integrals, Phys. Lett. B435 (1998) 350, hep-th/9804199.

[13] W. Krauth and M. Staudacher, Eigenvalue Distributions in Yang-Mills Integrals, Phys. Lett. B453 (1999) 253, hep-th/9902113.

[14] H. Aoki, S. Iso, H. Kawai, Y. Kitazawa and T. Tada, Space-Time Structures from IIB Matrix Model, Prog. Theor. Phys. 99 (1998) 713, hep-th/9802085.

[15] T. Hotta, J. Nishimura, A. Tsuchiya, Dynamical Aspects of Large $N$ Reduced Models, Nucl. Phys. B545 (1999) 543, hep-th/9811220.

[16] M. B. Green and M. Gutperle, D-Particle Bound States and the D-Instanton Measure, JHEP 9801 (1998) 005, hep-th/9711107.

[17] G. Moore, N. Nekrasov and S. Shatashvili, D-particle bound states and generalized instantons, hep-th/9803265.

[18] M. Fukuma, H. Kawai, Y. Kitazawa and A. Tsuchiya, String Field Theory from IIB Matrix Model, Nucl. Phys. B510 (1998) 158, hep-th 9705128.

[19] A. Ambjorn, K. Anagnostopoulos, W. Bietenholz, T. Hotta and J. Nishimura, Monte Carlo Studies of SUSY Large N Reduced Models, Poster presented by J. Nishimura at Strings '99. 